\title{
Activación de vías redox en respuesta a cargas únicas de ejercicio
}

\author{
Redox pathways activation in response to single bouts of exercise
}

\section{Alvarado-Espinoza, Brenda Linda ${ }^{1}$, Carrasco-Legleu, Claudia Esther ${ }^{1}$, González-Rodríguez, Everardo², Reza-López, Sandra Alicia²}

${ }^{1}$ Universidad Autónoma de Chihuahua, Facultad de Ciencias de la Cultura Física. Circuito Universitario Campus II, Fracc. Campo Bello, 31124, Chihuahua México. Tel. +52 6141589900 ext. 4639.

2Universidad Autónoma de Chihuahua, Facultad de Medicina y Ciencias Biomédicas. Circuito Universitario Campus II , Fracc. Campo Bello, 31109, Chihuahua México. Tel. +52 6142386030.

CORRESPONDENCIA

\section{Sandra Alicia Reza-López:}

sreza@uach.mx

Universidad Autónoma de Chihuahua, Facultad de Medicina y Ciencias Biomédicas.

\section{CÓMO CITAR}

Alvarado, B. L., Carrasco, C. E., González, E. y Reza, S. A. (2020). Activación de vías redox en respuesta a cargas únicas de ejercicio. Revista de Ciencias del Ejercicio, 15 (1), pp. 31-47. Recuperado de: revistafod.uanl.mx

\section{RESUMEN}

La actividad física insuficiente se asocia con el desarrollo de enfermedades crónicas no transmisibles. Promover la práctica de ejercicio se vuelve una estrategia importante para contribuir en su prevención y tratamiento. Las reacciones redox se presentan como parte de la respuesta metabólica al ejercicio. Se desarrolló una revisión sistemática en seis bases de datos con el objetivo de identificar estudios que reportaran biomarcadores redox asociados con cargas únicas de ejercicio. Se localizaron nueve publicaciones de los últimos 10 años, en idioma inglés, que cumplían con los criterios de elegibilidad (estudios originales, en humanos, que incluyeran mecanismos/biomarcadores redox). La mayoría de los biomarcadores reportados fueron proteínas y ARNm, analizados en sangre y/o músculo. Los cambios en marcadores se observaron mayormente dentro de las tres primeras horas después de cargas únicas de ejercicio físico. Individuos con actividad sedentaria, obesidad o de edad mayor a los 50 años, mostraron mayores niveles de productos de oxidación después de la carga de ejercicio que sujetos entrenados, activos o de menor edad. Los estudios sugieren que en sujetos entrenados se requiere valorar los cambios en biomarcadores redox mínimo a las 3 horas post-carga para detectar diferencias en las respuestas, las cuales son mayores después de cargas de duración larga e intensa. El estudio de cambios en biomarcadores redox ante ejercicio físico contribuye a una mayor comprensión de la respuesta fisiológica generada. Esto sería de utilidad para ajustar el entrenamiento para promover los beneficios del ejercicio y prevenir el potencial daño de un estrés oxidativo crónico.

Palabras clave: ejercicio, redox, biomarcadores, señalización.

\section{ABSTRACT}

Insufficient physical activity is associated with the development of non-communicable chronic diseases. Promoting exercise practice becomes an important strategy to contribute to their prevention and treatment. Redox reactions are elicited as part of the metabolic response to single bouts of exercise. A systematic review was conducted across six data bases aimed to identify studies reporting redox biomarkers associated with exercise. Nine publications from the last ten years were found in English language, fulfilling the eligibility criteria (original studies, in humans, that included redox mechanisms/biomarkers). Most of the reported biomarkers were proteins and mRNA, analyzed in blood and/or muscle. Biomarker changes were mainly observed within the first 3 hours after a single bout of physical exercise. Individuals with sedentary activity, obesity or older than 50 years of age, showed higher levels of oxidized products after the exercise bout, than trained, active or younger subjects. Studies suggest that in trained individuals there is the need to evaluate the redox biomarker changes at least 3 hours post-exercise to detect response differences, which are higher after long and intense bouts. The study of redox biomarker changes in physical exercise contributes to gain insight on the physiological response generated. This would be useful to adjust physical training to promote exercise benefits and to prevent the potential of chronic oxidative stress damage.

Key words: exercise, redox, biomarkers, signaling. 
Introducción

En los últimos 10 años, el número de casos de enfermedades no transmisibles han aumentado hasta $51-80$ por cada 100,000 habitantes en México y su aumento a través de los años se ha atribuido a condiciones ambientales y/o hábitos tales como estrés, tabaquismo, dieta de baja calidad y sedentarismo (Córdova-Villalobos et al., 2008; Evia y Roberto, 2018). Estudios han demostrado que la inactividad física se asocia con aproximadamente un $6-10 \%$ de las principales enfermedades crónicas no transmisibles, lo que contribuye en un $9 \%$ en la mortalidad prematura a nivel global (Lee et al., 2012; Lim et al., 2012; Pruss-Ustun, Wolf, Corvalán, Bos y Neira, 2016).

El ejercicio es un factor que representa una estrategia para prevenir las enfermedades crónicas no transmisibles (Schieber y Chandel, 2014). Con la práctica del ejercicio físico, se desarrolla una adaptación a la serie de cambios fisiológicos que se suscitan. Dentro de las respuestas generadas se encuentran aquellas de índole metabólico, circulatorio, cardiaco, respiratorio y hemático (Ferné-Vaquero, Serratosa-Fernández y López-Chicharro, 2006; Fernández-Vaquero, López-Chicharro, Lucía-Mulas y Pérez-Ruiz, 2006; Firman, 2015; Gabriel y Zierath, 2017; López-Chicharro y Lucía-Mulas, 2006; López-Chicharro y Pérez-Ruiz, 2006; Pérez-Ruiz, Córdova-Martínez, López-Chicharro, y Lucía-Mulas, 2006). Como parte de los procesos de utilización de sustratos energéticos ante una carga de ejercicio, se generan una serie de sustancias reactivas, dentro de las que se destacan el ion superóxido, peróxido de hidrógeno, ion hidroxilo, peroxinitrito, peroxilo y ácido hipocloroso, siendo las especies reactivas de oxígeno (ERO) las más importantes biológicamente (Ac- cattato et al., 2017; Trayhurn, Wang y Wood, 2018); sin embargo, el organismo neutraliza estas sustancias mediante la activación de sistemas antioxidantes. Durante el ejercicio prolongado, por ejemplo en el entrenamiento de resistencia, se han identificado mecanismos alternativos a través de los cuales se modula el equilibrio de las especies oxidantes y antioxidantes. Este podría ser un mecanismo importante, considerando que al sobrepasar el umbral del organismo para soportar el aumento de ERO, inicia una reacción en cadena que puede conducir a un daño a nivel tisular, como producto de la transformación (i.e. oxidación) de proteínas, lípidos y ácidos nucleicos, lo cual se identifica como estrés oxidativo [EO] (Berlett y Stadtman, 1997; Boufleur et al., 2011; Cobley, Close, Bailey y Davison, 2017).

En los estudios sobre la relación del EO con el ejercicio, se han analizado una gran diversidad de biomarcadores. Es posible determinar las ERO tales como el ion superóxido o el peróxido de hidrógeno (H2O2), o bien agentes antioxidantes, ya sea endógenos, como las enzimas superóxido dismutasa (SOD), catalasa (CAT), ciclooxigenasa (COX) y paraoxonasa (PON), o exógenos como la vitamina C o coenzima Q10, que permiten valorar la capacidad homeostática del individuo, en términos de equilibrio oxidante/antioxidante (Corrales y Ariza, 2012; Fernández, Da Silva-Grigoletto, y Túnez-Fiñana, 2009; Li, 1995; Mittler, 2002; M. G. Nikolaidis y Jamurtas, 2009; Viada Pupo, Robles, Reyna y Marrero, 2017). Algunos biomarcadores son indicadores indirectos de un desequilibrio entre agentes oxidantes y antioxidantes. Entre ellos se encuentran productos de la oxidación lipídica, como el malondialdehído, proteica como las proteínas carboniladas o nucleotídica, 
como la 8-hidroxi-2'-deoxiguanosina o 8-OHdG (Berlett y Stadtman, 1997; M. Nikolaidis et al., 2015; Schieber y Chandel, 2014; Webb, Hughes, Thomas y Morris, 2017) así como los moduladores de las enzimas antioxidantes, como son citocinas (i.e. adiponectina), o factores de transcripción, como el factor nuclear kappa B (NF-OB) y el factor nuclear eritroide 2-relacionado al factor 2 (Nrf2) que regulan la expresión génica de SOD o CAT (Miller et al., 2012; Pall y Levine, 2015; Sakurai et al., 2017). Sin embargo, se han obtenido resultados contradictorios en el proceso de determinación cuantitativa o cualitativa de estos biomarcadores, razón por la cual se vuelve trascendente analizar a detenimiento estudios que los incluyan.

La regulación de la expresión génica de biomarcadores relevantes, posibilita cambios funcionales durante el proceso de acondicionamiento. Esta expresión a su vez es modulada por procesos de transcripción, procesamiento de ácido ribonucleico (ARN), traducción de proteínas, estabilización proteica, así como modificaciones postraduccionales (Mougios, 2006b, 2006a; Rivera-Brown y Frontera, 2012), en este sentido, las vías involucradas son numerosas y pueden variar por el tipo de ejercicio, entre otros factores.

El propósito de este estudio fue identificar biomarcadores redox que se modifican en respuesta a cargas únicas de ejercicio en adultos.

\section{Método}

Fuentes de información
Se desarrolló una búsqueda de información en febrero de 2019 en las bases de datos PubMed, Scielo, Redalyc, EBSCO, Web de la Ciencia y SCOPUS.

La revisión se llevó a cabo siguiendo los lineamientos de PRISMA (del inglés Prefered Reporting Items for Systematic Reviews and Meta-Analyses). En las bases PubMed y Scielo se utilizaron los términos "Redox signaling" y "Exercise". En las bases Redalyc, EBSCO, Web de la Ciencia y SCOPUS se utilizaron los mismos términos, además de su traducción en español. En todas las bases se aplicó el operador boleano "AND". Solamente se incluyeron artículos originales escritos en idiomas inglés, español, portugués o francés, publicados en los últimos 10 años. No hubo limitaciones en términos de su localización geográfica. Se solicitaron textos completos libres en PubMed y de acceso abierto en Web de la Ciencia. En cuestión de restricción de categorías o áreas de interés, en caso de la base Redalyc se restringieron a medicina, salud multidisciplinaria, biología y química; en relación a Web de la Ciencia se eligieron bioquímica, biología molecular, fisiología, endocrinología metabolismo, biología celular, ciencias del deporte y química medicinal; en la búsqueda de SCOPUS se incluyeron bioquímica, genética y biología molecular, y medicina. En EBSCO se utilizaron los siguientes términos: activación, adulto, metabolismo antioxidantes, apoptosis, bioquímica y biología molecular, biomarcadores, enfermedades cardiovasculares, biología celular, citocinas, desarrollo 
y progresión, ejercicio, femenino, radicales libres, expresión génica, aspectos génicos, aspectos de salud, homeostasis, humanos, hipoxia, isquemia, cinasas, lípidos, masculino, medicina, investigación y experimental, metabolismo, mediana edad, enfermedad del metabolismo mitocondrial, endocrinología y metabolismo, enzimas, músculos, nf-kappa-b, óxido nítrico, oxidación-reducción, estrés oxidativo, fosforilación, aspectos fisiológicos, fisiología, proteínas, especies reactivas de oxígeno, artículo de investigación, factores de riesgo, transducción de señales, músculo esquelético.

\section{Criterios de eligibilidad}

Se incluyeron los estudios que cumplieron con los siguientes criterios: (1) artículos originales que reportan estudios controlados aleatorizados/no aleatorizados; (2) estudios desarrollados con sujetos humanos; (3) estudios que asociaban de una manera directa el ejercicio físico con mecanismos/ biomarcadores de señalización redox.

\section{Resultados}

En la figura 1 se muesta el proceso de selección de los artículos incluídos en esta revisión. De los 9 artículos elegidos, cuatro corresponden a estudios controlados aleatorizados, cuatro fueron ensayos cruzados y uno, transversal. En cuatro estudios se incluyeron sujetos estadounidenses; mientras que el resto involucraron sujetos españoles, ingleses, iraníes, australianos o suizos (un estudio en cada población). En todos los estudios se utilizaron muestras de sangre venosa como fuente biológica de análisis, en cuatro se usó tejido muscular y en uno se utilizó una muestra de orina. Las características principales de los estudios, momentos de toma de muestra y hallazgos más relevantes se describen en la Tabla 1.

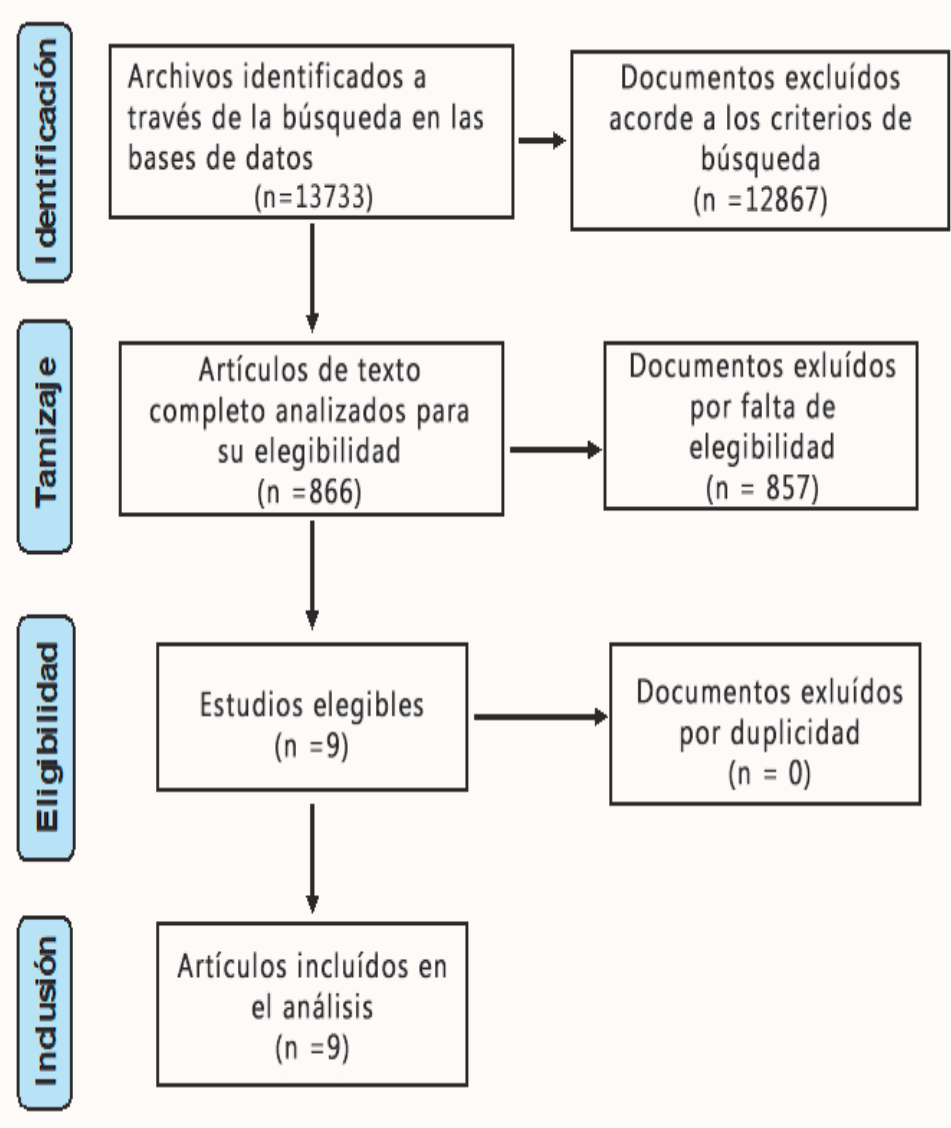

Figura 1. Diagrama que representa gráficamente el flujo de las citas analizadas en el transcurso de la revisión sistemática. En la primera etapa se describen los archivos encontrados con los términos de interés, y los excluídos al agregar los criterios de búsqueda en cada base de datos.

En la segunda etapa se distinguen aquellos excluídos por no reunir los criterios de elegibilidad. En la tercera etapa se muestra que no se requirió eliminar algún documento por duplicidad, por lo que los mismos nueve documentos se incluyeron en el análisis. 
Tabla 1. Estudios que reportan biomarcadores redox en respuesta a cargas únicas de ejercicio.

\begin{tabular}{|c|c|c|c|c|c|c|}
\hline $\begin{array}{c}\text { Lugar de } \\
\text { publicación }\end{array}$ & $\begin{array}{l}\text { Diseño del } \\
\text { estudio }\end{array}$ & Sujetos & $\begin{array}{c}\text { Intervención/ } \\
\text { Cargas de ejercicio }\end{array}$ & $\begin{array}{l}\text { Muestra } \\
\text { biológica }\end{array}$ & $\begin{array}{c}\text { Tiempo de colecta de } \\
\text { muestra }\end{array}$ & Resultados vinculados con biomarcadores redox \\
\hline $\begin{array}{l}\text { Irán } \\
\text { (Abdizadeh, } \\
\text { Jafari y } \\
\text { Armanfar, } \\
\text { 2015) }\end{array}$ & A & $\begin{array}{l}20 \text { escaladores } \\
\text { de montaña } \\
\text { varones. Edad: } \\
24.8 \pm 2.8 \text { años. }\end{array}$ & $\begin{array}{l}\text { Intervención: Suplementación } \\
\text { con Coenzima Q10 a } 5 \\
\mathrm{mg} / \mathrm{kg} / \mathrm{día} \\
\text { Ejercicio: } 30 \mathrm{~min} \text { corriendo en } \\
\text { banda al } 65 \% \text { de } \mathrm{VO}_{2} \text { máx, } \\
\text { inclinación de }-15 \%\end{array}$ & Sangre & $\begin{array}{l}\text { En reposo, después de } 2 \\
\text { semanas de la } \\
\text { suplementación, y } 18 \text { y } 24 \\
\text { horas post-carga }\end{array}$ & $\begin{array}{l}\text { Se aumentó significativamente la AAT después de } 2 \text { semanas de } \\
\text { intervención y post-carga. No hubo cambios significativos con } \\
\text { placebo. } \\
\text { MDA se mantuvo igual en el grupo de intervención en todos los } \\
\text { tiempos. Después del ejercicio, los niveles en el grupo placebo fueron } \\
\text { mayores respecto a los que consumieron Q10. }\end{array}$ \\
\hline $\begin{array}{l}\text { España } \\
\text { (Casuso et al., } \\
2017 a \text { ) }\end{array}$ & B & $\begin{array}{l}9 \text { nadadores } \\
\text { varones } \\
\text { entrenados } \\
\text { Edad: } 23 \pm 3 \\
\text { años. }\end{array}$ & $\begin{array}{l}\text { a.10 cargas de ejercicio de } 200 \\
\mathrm{~m} \text { a una intensidad alta } \\
\text { (ensayo HIHVT), constante } \\
\text { b.10 cargas de ejercicio de } 50 \\
\mathrm{~m} \text { a la mayor velocidad } \\
\text { posible (ensayo SIT) } \\
\text { Las intervenciones se } \\
\text { realizaron en orden aleatorio, } \\
\text { con } 7 \text { días de diferencia entre } \\
\text { ellas }\end{array}$ & $\begin{array}{l}\text { Sangre y } \\
\text { tejido } \\
\text { muscular } \\
\text { (m.triceps } \\
\text { bracci) }\end{array}$ & $\begin{array}{l}\text { En reposo, } 3 \min \text { y } 3 \\
\text { horas } \\
\text { (sangre); } \\
\text { En repost-cargas } \\
\text { horas } \\
\text { (músculo) }\end{array}$ & $\begin{array}{l}\text { SIT } \\
\text { Concentración sérica de hidroperóxidos y PGC-1 } \alpha \text { muscular, así como } \\
\text { niveles de ARNm de SIRT1, SIRT3, SOD2, COX-1, NRF1 y TFAM en } \\
\text { músculo, no cambiaron en respuesta a ejercicio. } \\
\text { Se observó activación de AMPK muscular a los } 5 \text { min post-carga. } \\
\text { ARNm de PGC-1 } \alpha \text { en músculo aumentó mayormente a las } 3 \text { hr; } \\
\text { el de VEGFA aumentó } 2 \text { veces a los } 5 \text { min post-carga y disminuyó a las } \\
3 \text { hr. } \\
\text { HIHVT } \\
\text { La concentración de hidroperóxidos séricos y ARNm de SIRT1 en } \\
\text { músculo no cambió. } \\
\text { Se activó AMPK muscular mayormente a las } 3 \text { hr. } \\
\text { Otros ARNm en músculo: } \\
\text { PGC-1 } \alpha \text { sólo aumentó a las } 3 \mathrm{hr} \text {-así como su nivel proteico- } \\
\text { SIRT3 sólo aumentó a las } 3 \mathrm{hr} \text { post-caga } \\
\text { SOD2 disminuyó a los } 5 \text { min post-carga } \\
\text { VEGFA sólo aumentó } 4 \text { veces a las } 3 \mathrm{hr} \text {. } \\
\text { COX-1 sólo aumentó } 4 \text { veces a las } 3 \mathrm{hr} \text {. } \\
\text { NRF1 y TFAM disminuyeron a } 5 \text { min post-carga }\end{array}$ \\
\hline $\begin{array}{l}\text { Reino Unido } \\
\text { (Constantin- } \\
\text { Teodosiu, } \\
\text { Constantin, }\end{array}$ & A & $\begin{array}{l}6 \text { hombres no } \\
\text { entrenados } \\
\text { Edad: } 22 \pm 0.6 \\
\text { años. }\end{array}$ & $\begin{array}{l}\text { Intervención: Administración } \\
\text { de dicloroacetato, DCA (50 } \\
\mathrm{mg} / \mathrm{kg} \text { peso) bajo dieta alta en } \\
\text { grasas }\end{array}$ & $\begin{array}{l}\text { Sangre y } \\
\text { músculo } \\
\text { (vastus } \\
\text { lateralis) }\end{array}$ & $\begin{array}{l}\text { En reposo, } 10 \text { y } 60 \mathrm{~min} \\
\text { post-carga (músculo); en } \\
\text { reposo, } 10,20,30,40,50 \\
\text { y } 60 \mathrm{~min} \text { post-carga }\end{array}$ & $\begin{array}{l}\text { Dieta control } \\
\text { ARNm en músculo: } \\
\text { AKT1, PPAR } \alpha \text {, PPARס, FOXO3, IRS1, PPM2C, PDP2, PGC-1 } \alpha \text { y PDK no } \\
\text { se modificaron por dieta o ejercicio. }\end{array}$ \\
\hline
\end{tabular}




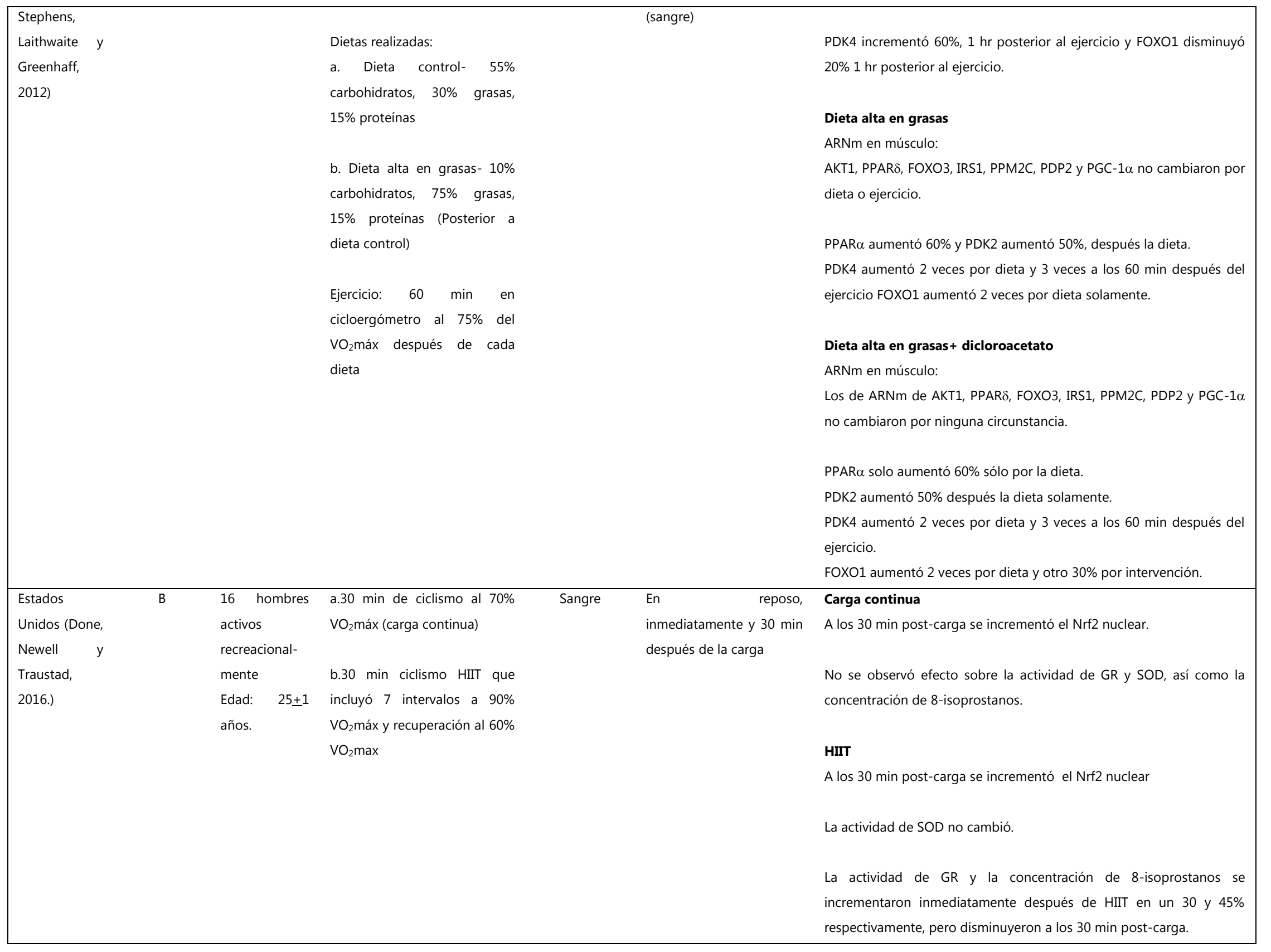




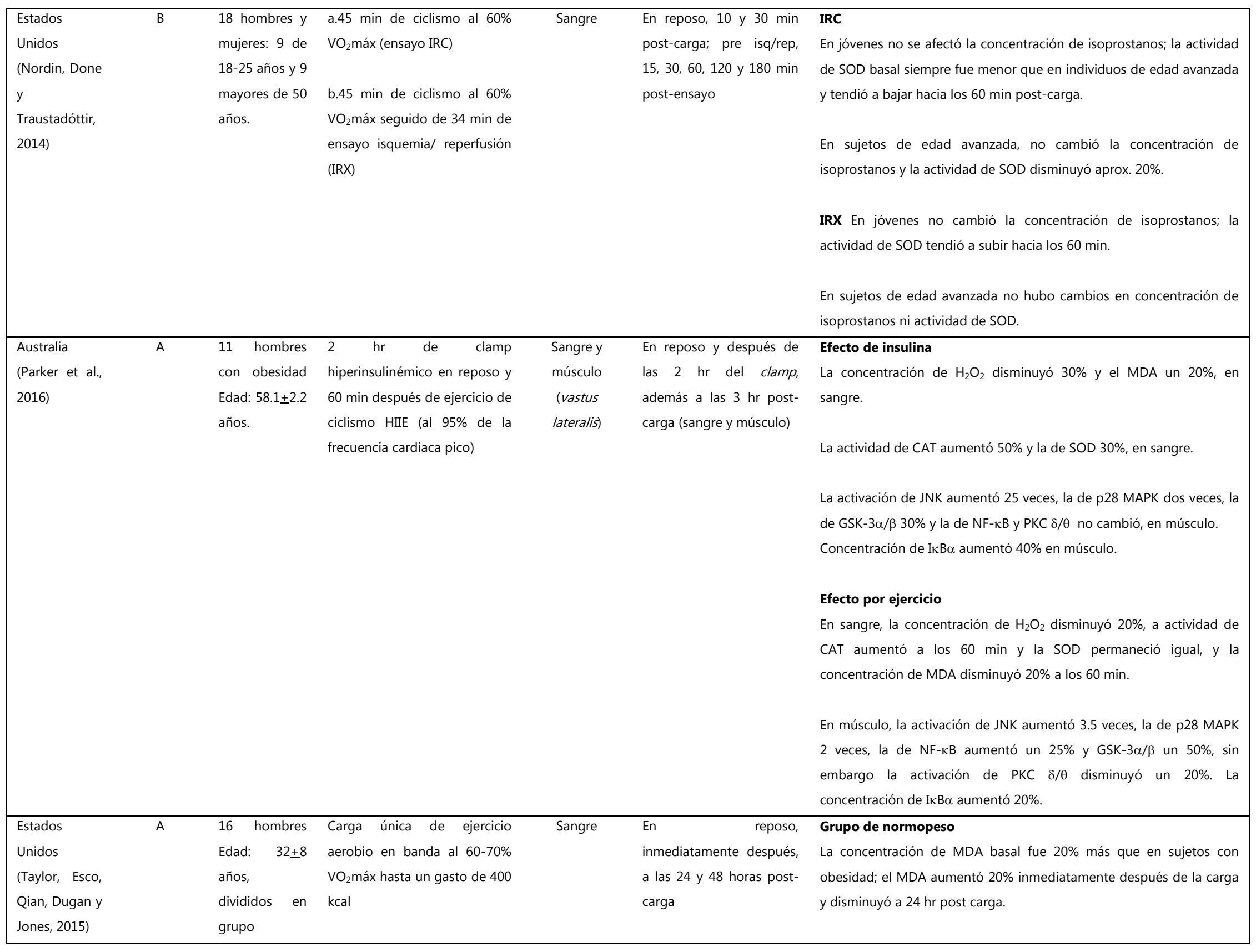




\begin{tabular}{|c|c|c|c|c|c|c|}
\hline & & $\begin{array}{l}\text { normopeso y } \\
\text { grupo con } \\
\text { obesidad }\end{array}$ & & & & $\begin{array}{l}\text { La actividad y concentración de PON1 no se modificaron con el } \\
\text { tiempo. } \\
\text { Grupo de obesidad, GO } \\
\text { El MDA aumentó } 20 \% \text { justo después de la carga y disminuyó hasta las } \\
48 \mathrm{hr} \text {. } \\
\text { La actividad de PON1 aumentó } 10 \% \text { inmediatamente después de la } \\
\text { carga y siempre fue menor que en grupo normopeso. } \\
\text { La concentración de PON1 no se modificó con el tiempo y siempre } \\
\text { fue menor en todos los momentos respecto al grupo normopeso. }\end{array}$ \\
\hline $\begin{array}{l}\text { Estados } \\
\text { Unidos } \\
\text { (Traustadóttir } \\
\text { et al., 2012) }\end{array}$ & C & $\begin{array}{l}72 \text { hombres y } \\
\text { mujeres } \\
\text { acondiciona- } \\
\text { dos y } 35 \text { no } \\
\text { acondiciona- } \\
\text { dos) de } 60-80 \\
\text { años. }\end{array}$ & $\begin{array}{l}\mathrm{VO}_{2} \text { max y potencia máxima en } \\
\text { piernas a } 50,60,70 \% \text { de } 1 \text { - } \\
\text { repetición máxima }\end{array}$ & Sangre y orina & $\begin{array}{l}\text { En reposo y } 15,30,60, \\
120,180 \text { y } 240 \mathrm{~min} \text { post } \\
\text { isq/rep en reposo; pool } \\
\text { de orina de } 5 \text { días post- } \\
\text { carga (antes de la isq/rep) }\end{array}$ & $\begin{array}{l}\text { Sujetos con acondicionamiento } \\
\text { En ningún marcador se apreciaron cambios con el ejercicio. } \\
\text { Sujetos sin acondicionamiento } \\
\text { Isoprostanos séricos, capacidad antioxidante total y relación } \\
\text { GSH/GSSG en sangre, no presentaron ningún cambio debido al } \\
\text { ejercicio. } \\
\text { El 8-OHdG, 8-oxo-G e isoprostanos urinarios no presentaron cambios } \\
\text { debido a la carga pero siempre fueron mayores que en sujetos } \\
\text { acondicionados. }\end{array}$ \\
\hline $\begin{array}{l}\text { Suiza (Wang, } \\
\text { Mascher, } \\
\text { Psilander, } \\
\text { Blomstrand y } \\
\text { Sahlin, 2011) }\end{array}$ & B & $\begin{array}{l}10 \text { ( } 7 \text { hombres } \\
\text { y } 3 \text { mujeres) } \\
\text { activos } \\
\text { recreacional- } \\
\text { mente. Edad: } \\
26 \pm 1.2 \text { años. }\end{array}$ & $\begin{array}{l}\text { a.60 min de ciclismo al } 65 \% \\
\mathrm{VO}_{2} \text { max (protocolo } \mathrm{E} \text { ) } \\
\text { b. } 60 \text { min de ciclismo al } 65 \% \\
\mathrm{VO}_{2} \mathrm{max}+15 \text { min reposo }+6 \\
\text { sets de press de pierna al } 70- \\
80 \% \text { de } 1 \mathrm{RM} \text { en } 25 \mathrm{~min} \\
\text { (protocolo ER) }\end{array}$ & $\begin{array}{c}\text { Sangre y } \\
\text { músculo } \\
\text { (entre rótula y } \\
\text { espina iliaca) }\end{array}$ & $\begin{array}{l}\text { En reposo, } 1 \text { y } 3 \text { hr post- } \\
\text { carga (sangre y músculo) }\end{array}$ & 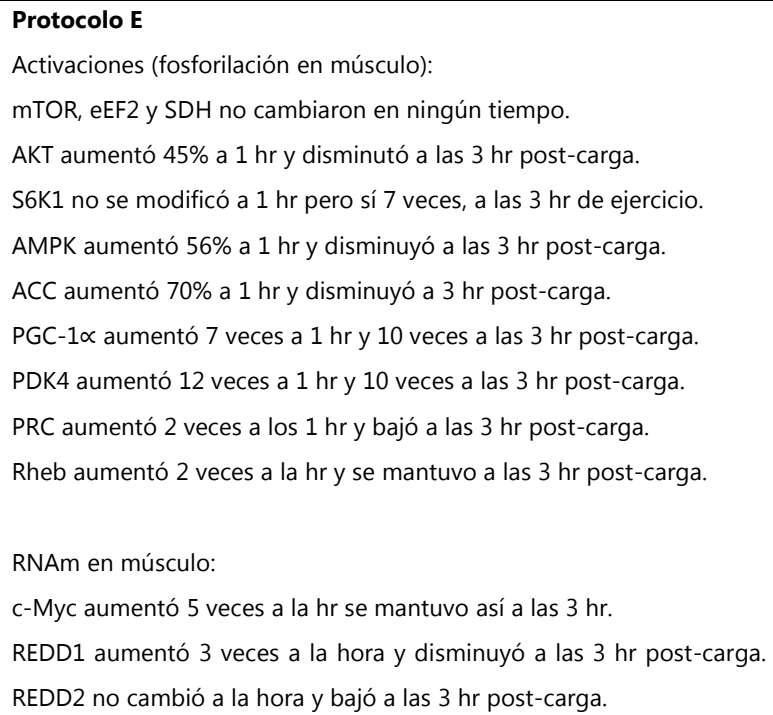 \\
\hline
\end{tabular}




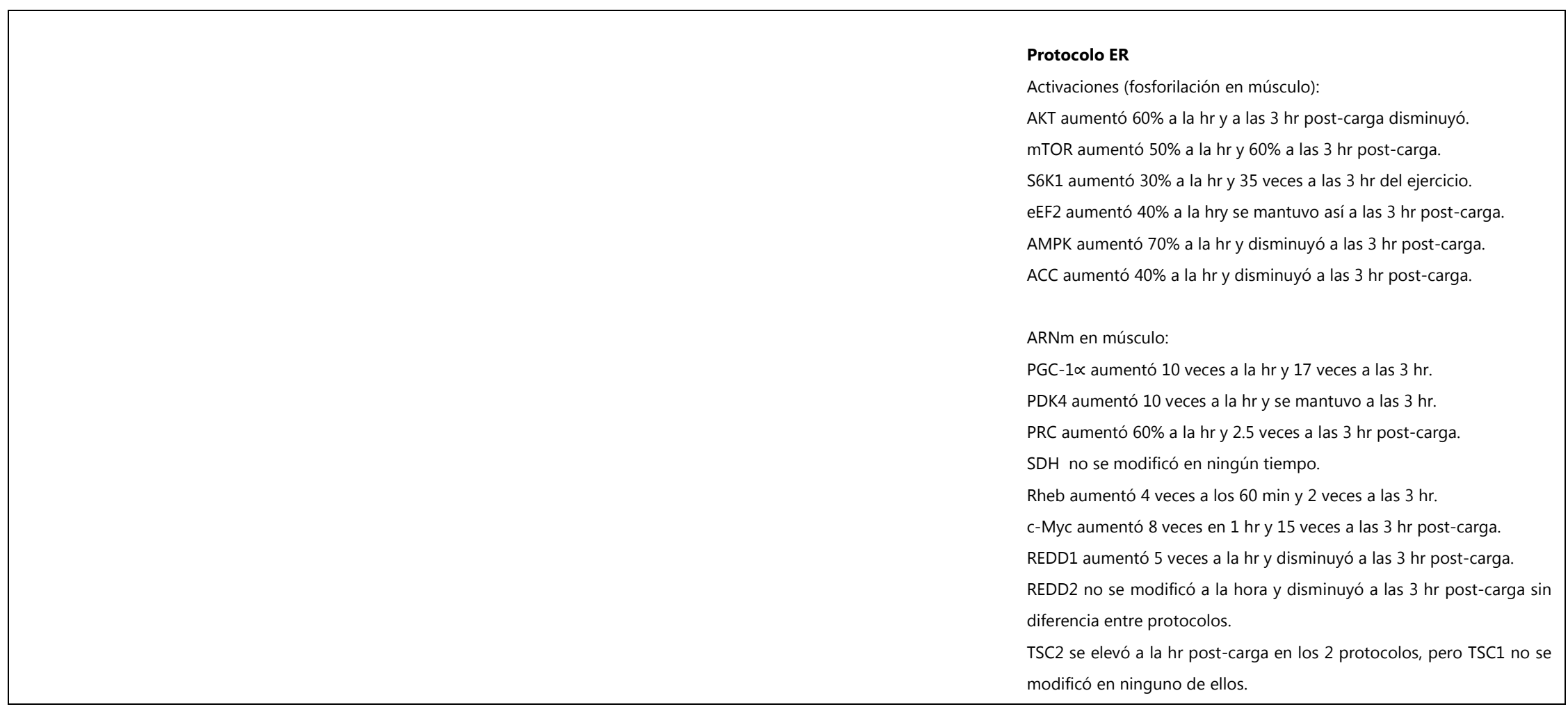

Diseño del estudio (A) Ensayo controlado no aleatorizado, (B) Ensayo cruzado y (C) Transversal. Significado de acrónimos: 8-OHdG: 8-hidroxi-2'deoxiguanosina; 8-oxo-G: 8-oxoguanina; AAT: actividad antioxidante total; ACC: acetil-CoA carboxilasa; AKT: serin-treonin proteincinasa; AMPK: proteincinasa dependiente de AMP; ARNm: ácido ribonucleico mensajero; CAT: catalasa; c-Myc: gen del protooncogen Myc; COX-1: ciclooxigenasa 1; DCA: dicloroacetato; eEF2: factor de elongación 2 diftamida; FOXO-1/3: del inglés forkhead box protein O1/3;

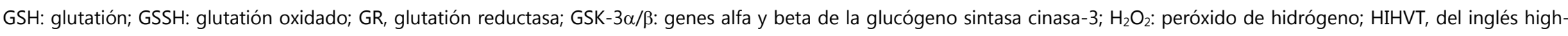

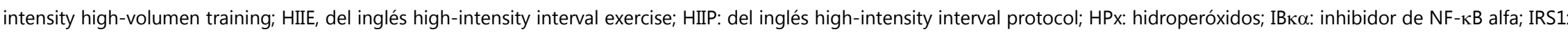
sustrato 1 del receptor de insulina; JNK: cinasa c-JUN N-terminal; MAPK: del inglés mitogen-activated protein kinase; MDA: malondialdehído; mTOR: blanco mamífero de la rapamicina; NF- $\mathrm{KB}$ : factor nuclear potenciador de las cadenas ligeras kappa de las células B; NRF1/2: factor 1/2 relacionado con el factor nuclear eritroide; PDK2/4: piruvato deshidrogenasa cinasa 2/4; PDP2: piruvato deshidrogenasa fosfatasa; PGC-1 $\alpha$ : coactivador 1 alfa del receptor activado proliferador peroxisomal gamma; PKC: proteincinasa; PON1: paraoxonasa 1; PPAR $\alpha / \delta$ : receptor activado por proliferadores peroxisomales alfa/delta; PPM2C: subunidad 1 de la PDP2; PRC: proteína reguladora de citocinesis; REDD1/2: del inglés regulated in development and DNA damage response 1/2; Rheb: homólogo de RAS enriquecido en cerebro; SAPK: proteína cinasa activada por estrés; SDH: succinato deshidrogenasa; SGK1: cinasa 1 inducida por suero y glucocorticoides; SIRT1/3: sirtuina 1/3; SIT: del inglés sprint interval training; SOD2: superóxido dismutasa 2; TFAM: factor de transcripción A mitocondrial; TSC1/2: complejo de esclerosis tuberosa 1/2; VEGFA: factor de crecimiento endotelial vascular; $\mathrm{VO}_{2}$ máx: Consumo máximo de oxígeno. 
Como se observa en la tabla 1 , los artículos describen el efecto de intervenciones diversas en las cuales se aplicó una carga única de ejercicio aerobio, y en dos de ellos de manera adicional, se incluían ensayos con ejercicio de fuerza (Traustadóttir et al., 2012; Wang et al., 2011). Las cargas aplicadas fueron al $60 \%$ o más del consumo máximo de oxígeno, siendo los más frecuentes aquellos realizados al $65 \%$ del VO2máx. En torno a los ejercicios de fuerza, éstos fueron realizados al $50 \%-80 \%$ de una repetición máxima (1-RM).

Los tiempos de colecta de muestra después de la aplicación de la carga de ejercicio fueron variables: inmediatamente después de la aplicación del ejercicio (dentro de los primeros 5 minutos) en cuatro estudios, a los 10 minutos en tres estudios, $30 \mathrm{mi}-$ nutos en tres estudios, 60 minutos en tres estudios y a los 180 minutos post-carga en cuatro estudios. Los momentos en los que se observaron más cambios en los marcadores fueron en los primeros $180 \mathrm{~min}$ post-carga, siendo sangre venosa y tejido muscular las muestras más utilizadas. La respuesta aguda al ejercicio mostró una mayor diferencia a las 3 horas post-carga.

En la literatura revisada, se identificó una cantidad variable de biomarcadores. Los más utilizados fueron de naturaleza proteica, para analizar su actividad o concentración, y nucleotídica, para determinar cambios en la expresión génica. Se pudieron identificar 12 biomarcadores analizados en sangre. Estos incluían actividad antioxidante total, agentes reactivos oxidantes, productos de oxidación lipídica, agentes antioxidantes y reguladores de la expresión de agen- tes oxidantes. La investigación que involucró orina como muestra, incluyó el análisis de 3 biomarcadores incluyendo productos de oxidación de lípidos y de ácido desoxirribonucleico (ADN). Finalmente, en muestras de tejido muscular, se analizaron 32 biomarcadores proteicos -analizados en términos de concentración o activación después del ejercicio- o ARNm. Las funciones de las sustancias involucraban catálisis, señalización intracelular y modulación de la expresión génica. En términos generales, los biomarcadores más utilizados fueron los relativos a la oxidación lipídica (por los productos que generan), mientras que marcadores de oxidación de otras moléculas han sido menos estudiados, en relación a cargas únicas de ejercicio. Por otro lado, los antioxidantes mayormente analizados son aquellos de carácter enzimático, ya sea su actividad, concentración o diferencias de expresión post-carga. Cabe señalar que siete estudios consideran otras mediciones bioquímicas. Sin embargo, en la presente revisión se incluyen solamente los marcadores de EO/ señalización redox.

\section{Discusión}

Los estudios que se incluyen en esta revisión analizan los cambios en concentración, actividad o expresión de biomarcadores redox, así como mecanismos de señalización. Diversos estímulos pueden inducir éstos últimos procesos, tales como la oxidación de nutrientes, el contacto con contaminantes o el ejercicio físico (Buresh \& Berg, 2015; Fernández et al., 2009; M. Nikolaidis et al., 2012). Se ha reportado que acorde a la duración del ejercicio, su intensidad, la estimulación de fibras musculares o 
sus costos funcionales, se inducen distintas vías de señalización redox. Bajo un estímulo suficientemente intenso y constante, ésto puede llevar al desarrollo de estrés oxidativo. A continuación se mencionan distintas condiciones, que se encontraron influyen en los marcadores y vías utilizadas, como respuesta aguda al ejercicio.

Dentro de los agentes oxidantes identificados se encuentraron los peróxidos, específicamente $\mathrm{H} 2 \mathrm{O} 2$ e hidroxiperóxidos. Sus valores decrecieron conforme se incrementó el tiempo post-carga aerobia. Sin embargo, en sujetos entrenados aparentemente no cambiaron los niveles de hidroperóxidos aún con diferencias en intensidad de carga (Casuso et al., 2017b; Parker et al., 2016). Los productos de oxidación lipídica, como MDA e isoprostanos, se encontraron en mayor concentración en sangre de individuos no acondicionados o de edad avanzada y aumentaron inmediatamente después de aplicada una carga aerobia. Posteriormente tendieron a disminuir a los 60 min post-carga o más, dependiendo de la condición del sujeto. Los productos de oxidación de ADN en general se encuentraron en menores niveles en la orina de individuos entrenados que en no acondicionados, sin embargo no está claro si la aplicación de una carga de fuerza tiene efecto sobre los mismos, o si el tipo de muestra contribuye a interpretar esos hallazgos (Traustadóttir et al., 2012). La actividad enzimática antioxidante apreciable en sangre o tejido muscular fue variable acorde al marcador analizado, las condiciones del individuo y el tipo de intervención (Casuso et al., 2017b; Done et al., 2017; Nordin et al., 2014; Parker et al., 2016; Taylor et al., 2015; Wang et al., 2011). Las moléculas de señalización intracelular como AMPK se activaron dentro de los primeros 60 minutos después de aplicada la carga. Su función es impulsada cuando la carga de ejercicio es aerobia, y ésta es de un volumen mayor y constante (Casuso et al., 2017a; Parker et al., 2016; Wang et al., 2011). Respecto a los moduladores de la expresión génica, como tienen una participación variable en las distintas etapas de los mecanismos de señalización redox, las diferencias en su expresión también se ven afectadas acorde al marcador en particular. Cabe mencionar que dentro de los estudios analizados, las proteínas SIRT1, PDK2, AKT1, PPARD, PPARD, FOXO3, IRS1, PPM2C y SDH no mostraron evidencias de cambios en sus niveles de expresión en músculo, posterior al ejercicio aerobio y/o anaerobio (Casuso et al., 2017a; Constantin-Teodosiu et al., 2012; Wang et al., 2011).

Dependiendo de la naturaleza aerobia o anaerobia del ejercicio físico, es posible apreciar la activación de una serie de moléculas, básicamente con función efectora enzimática o de regulador de la expresión génica. Éstas desencadenan las vías de señalización que conducen a los efectos adaptativos del ejercicio. De acuerdo a los resultados de esta revisión, las vías impulsadas fueron aquellas dependientes de cinasas, como lo sugieren los cambios observados en la fosforilación de PGC-1D, que depende de AMPK y p38 MAPK (Casuso et al., 2017; Ferraro, Giammarioli, Chiandotto, Spoletini y Rosano, 2014; Martindales y Holbrook, 2002; Parker et al., 2016; Powers, Duarte, Kavazis, Talbert y Powers, 2009; Powers, Talbert, Adhihetty y 
Powers, 2011; Radak, Taylor, Ohno y Goto, 2001; Wang et al., 2011). En segunda instancia se identificó la vía de activación de mTOR. Como se encuentra reportado en otros estudios, la vía de mTOR se induce con la aplicación de ejercicio de fuerza (Perl, 2015; Tebay et al., 2015; Wang et al., 2011). Esto tiene relación con los hallazgos en los artículos analizados en la revisión sistemática (Traustadóttir et al., 2012; Wang et al., 2011), debido a que casi todos los biomarcadores identificados se vinculan a las vías estimuladas por cinasas como AMPK y MAPK, y en el caso de estos 2 reportes, donde se aplica de manera adicional una carga de fuerza, se describe la participación de los biomarcadores en la vía adicional de mTOR.

Los cambios encontrados en la respuesta aguda al ejercicio son multifactoriales, y las estrategias metodológicas en los estudios sin duda juegan un papel relevante en su hallazgo. Por un lado se tiene el aspecto del tiempo y el tipo de muestra a utilizar. Esto depende de qué aspecto del marcador se analizará, es decir, si se identificará su concentración, actividad o cambio en la expresión génica, ya que no todas las proteínas se expresan o secretan de igual manera. Adicionalmente es esencial considerar las características de los sujetos de estudio, tales como antecedentes de actividad física. Esto es debido a que las respuestas antioxidantes en sujetos entrenados por lo general son menores que en aquellos con actividad sedentaria o con obesidad. Por otro lado, se encuentra el perfil genético y temperatura corporal que influyen en la expresión y funcionalidad proteica. $Y$ en relación a la dieta, por ejemplo, se ha podido observar que el uso de agentes antioxidantes exógenos e insulina, disminuye la respuesta de biomarcadores oxidantes después de la aplicación de cargas únicas de ejercicio aerobio. Finalmente, las condiciones ambientales bajo las cuales se desarrolla la actividad física (como la temperatura ambiental o realizarlo en presencia de otros individuos), influyen en la magnitud de respuesta generada y los marcadores estimulados (Fernández et al., 2009).

\section{Conclusiones}

En el presente estudio se desarrolló una revisión de literatura de nueve documentos vinculados con la aplicación de cargas únicas de ejercicio en sujetos humanos, que permiten sentar las bases para estudios más dirigidos en torno a las respuestas celulares y moleculares dependientes de ejercicio. Después de su análisis se pudo encontrar una gran diversidad de biomarcadores redox modificables en respuesta a cargas únicas, entre los que se destacaron: la actividad antioxidante total, el oxidante $\mathrm{H} 2 \mathrm{O} 2$, los productos de oxidación lipídica isoprostanos y MDA, así como proteínas que participan en distintas etapas dentro de la señalización redox como AMPK, CAT, FOXO1, GR, JNK, MAPK, NFQB, NRF2, PDK, PGC-1D, SOD, entre otros.

El ejercicio es una práctica que lleva a la conservación de la salud y prevención de enfermedades crónicas no transmisibles, lo que en nuestro país tiene suma relevancia acorde a la gran incidencia de dichas enfermedades y su contribución en los índices de mortalidad. El ejercicio 
genera una respuesta en nuestro organismo que debe ser analizada adecuadamente desde el nivel molecular, para que las respuestas fisiológicas se vinculen exclusivamente a un mejor acondicionamiento corporal, sin comprometer el eventual funcionamiento del organismo por su carga excesiva.

\section{Agradecimientos}

Al Consejo Nacional de Ciencia y Tecnología, por la beca otorgada al primer autor.

\section{Referencias}

Fernández-Vaquero, A., Serratosa-Fernández, L., y López-Chicharro, J. (2006). Respuestas y adaptaciones cardiovasculares al ejercicio. En Fisiología del ejercicio (3era ed., pp. 321-356). Buenos Aires: Panamericana.

Abdizadeh, L., Jafari, A., y Armanfar, M. (2015). Effects of short-term coenzyme Q10 supplementation on markers of oxidative stress and inflammation after downhill running in male mountaineers. doi.org/10.1016/j.scispo.2015.02.012

Accattato, F., Greco, M., Pullano, S. A., Carè, I., Fiorillo, A. S., Pujia, A., ... Gulletta, E. (2017). Effects of acute physical exercise on oxidative stress and inflammatory status in young, sedentary obese subjects. PLoS ONE, 12(6), 1-13. doi. org/10.1371/journal.pone. 0178900

Berlett, B. S., y Stadtman, E. R. (1997). Protein oxidation in aging, disease, and oxidative stress. The Journal of Biological Chemistry, 272(33), 20313-20316. doi.org/10.1074/jbc.272.33.20313
Boufleur, J., Mariel, F., Terra, S., Sangoi, M., Santos, L., Pillon, R. y Alexandre, F. (2011). Response of oxidative stress and inflammatory biomarkers to a 12-week aerobic exercise training in women with metabolic syndrome. https://doi. org/10.1186/s40798-015-0011-2

Buresh, R., y Berg, K. (2015). A tutorial on oxidative stress and redox signaling with application to exercise and sedentariness. Sports Medicine-Open, 1(3), 1-9. doi.org/10.1186/s40798-014-0003-7

Casuso, R., Plaza-Díaz, J., Ruiz-Ojeda, F. J., Nimo, J., Ndido, C., Nordsborg, B... Huertas, J. R. (2017a). High-intensity high-volume swimming induces more robust signaling through PGC-1D and AMPK activation than sprint interval swimming in $\mathrm{m}$. triceps brachii. doi. org/10.1371/journal.pone.0185494

Cobley, J. N., Close, G. L., Bailey, D. M., y Davison, G. W. (2017). Exercise redox biochemistry: Conceptual, methodological and technical recommendations. Redox Biology, 12, 540-548. doi.org/10.1016/j.redox.2017.03.022

Constantin-Teodosiu, D., Constantin, D., Stephens, F., Laithwaite, D., y Greenhaff, P. L. (2012). The Role of FOXO and PPAR Transcription Factors in Diet-Mediated Inhibition of PDC Activation and Carbohydrate Oxidation During Exercise in Humans and the Role of Pharmacological Activation of PDC in Overriding These Changes. Diabetes, 61, 1017-1024. doi.org/10.2337/db11-0799

Córdova-Villalobos, J. A., Barriguete-Meléndez, J. A., Lara-Esqueda, A., Barquera, S., Rosas-Peralta, M., Hernández-Ávila, M., ... Aguilar-Salinas, C. A. 
(2008). Las enfermedades crónicas no transmisibles en México: sinopsis epidemiológica y prevención integral. Salud Pública de México, 50(5), 419-427. Recuperado de http://saludpublica.mx/index. php/spm/article/view/6847/8645

Corrales, L. C., y Ariza, M. M. M. (2012). Estrés oxidativo: origen, evolución y consecuencias de la toxicidad del oxígeno. Nova - Publicación Científica En Ciencias Biomédicas, 10, 213-225. Recuperado de http://www.scielo.org.co/pdf/nova/ v10n18/v10n18a08.pdf

Done, A. J., Newell, M. J., y Traustad, T. (2017). Effect of exercise intensity on Nrf2 signalling in young men. Free Radical Research, 51(6), 646-655. doi.org/10.1080/1 0715762.2017.1353689

Evia, B., y Roberto, J. (2018). México y el reto de las enfermedades crónicas no transmisibles. El laboratorio también juega un papel importante. Rev Latinoam Patol Clin Med Lab (Vol. 65). Recuperado de www.medigraphic.com/patologiaclinicawww.medigraphic.org.mxwww.medigraphic.org.mx

Fernández-Vaquero, A., López-Chicharro, J., Lucía-Mulas, A., y Pérez-Ruiz, M. (2006). Capacidad funcional aeróbica y anaeróbica. En Fisiología del ejercicio (3era ed., pp. 405-542). Madrid: Panamericana.

Fernández, J., Da Silva-Grigoletto, M. E., y Túnez-Fiñana, I. (2009). Estrés oxidativo inducido por el ejercicio. Revista Andaluza de Medicina Del Deporte, 1(3), 2008. Recuperado de http://www.redalyc.org/ pdf/3233/323327657004.pdf

Ferraro, E., Giammarioli, A. M., Chiandotto, S., Spoletini, I., y Rosano, G. (2014). Exer-
cise-Induced Skeletal Muscle Remodeling and Metabolic Adaptation: Redox Signaling and Role of Autophagy. Antioxidants \& Redox Signaling, 21(1), 154-157. doi. org/10.1089/ars.2013.5773

Firman, G. (2015). Fisiologia del ejercicio físico. Recuperado de https://vdocuments.mx/fisiologia-del-ejercicio-drguillermo-o-firman.html

Gabriel, B. M., y Zierath, J. R. (2017). Cell Metabolism The Limits of Exercise Physiology: From Performance to Health. doi.org/10.1016/j.cmet.2017.04.018

Lee, I.-M., Shiroma, E., Lobelo, F., Puscka, P., Blair, S., y Katzmarzyk, P. (2012). Impact of physical inactivity on the world's mayor non-communicable diseases. Lancet, 380(9838), 219-229. doi. org/10.1016/S0140-6736(12)61031-9

\section{Li, J. L. (1995). Exercise and Oxidative} Stress: Role of the cellular antioxidant systems.pdf. Exercise and Sport Sciences Reviews, 23(1), 135-166. Recuperado de https://journals.lww.com/acsm-essr/Citation/1995/00230/Exercise_and_Oxidative_Stress_Role_of_the.7.aspx

Li Ji, L. (2015). Redox signaling in skeletal muscle: role of aging and exercise. Adv Physiol Educ, 39, 352-359. doi. org/10.1152/advan.00106.2014

Lim, S. S., Vos, T., Flaxman, D., Danaei, G., Shibuya, K., Adair-Rohani, H., ... Andrews, K. (2012). A comparative risk assessment of burden of disease and injury attributable to 67 risk factors and risk factor clusters in 21 regions, 1990-2010: a systematic analysis for the Global Burden of Disease Study 2010. Lancet, 380(9859), 2224-2260. doi. org/10.1016/S0140-6736(12)61766-8 
López-Chicharro, J., y Lucía-Mulas, A. (2006). Respuestas y adaptaciones pulmonares al ejercicio. En Fisiología del ejercicio (3era ed., pp. 357-404). Buenos Aires: Panamericana.

López-Chicharro, J., y Pérez-Ruiz, M. (2006). Respuestas y adaptaciones de sistemas funcionales. En Fisiología del ejercicio (3era ed., pp. 543-592). Buenos Aires: Panamericana.

Martindale, J. L., y Holbroock, N. J. (2002). Cellular response to oxidative stress: signaling for suicide and survival. Journal of Cellular Physiology, 192, 1-15.

Miller, C. J., Gounder, S. S., Kannan, S., Goutam, K., Muthusamy, V. R., Firpo, M. A., ... Rajasekaran, N. S. (2012). Disruption of Nrf2/ARE signaling impairs antioxidant mechanisms and promotes cell degradation pathways in aged skeletal muscle. Biochimica et Biophysica Acta - Molecular Basis of Disease. doi.org/10.1016/j.bbadis.2012.02.007

Mittler, R. (2002). Oxidative stress, antioxidants and stress tolerance. Trends in Plant Science, 7(9), 405-410. doi.org/10.1016/ S1360-1385(02)02312-9

Mougios, V. (2006a). Effects of exercise on gene expression. In Exercise biochemistry (1era ed., pp. 227-236). Thessaloniki: Human Kinetics Publishers.

Mougios, V. (2006b). Integration of exercise metabolism. In Exercise biochemistry (1era ed., pp. 237-265). Thessaloniki: Human Kinetics Publishers.

Nikolaidis, M. G., \& Jamurtas, A. Z. (2009). Blood as a reactive species generator and redox status regulator during exercise. Archives of Biochemistry and Biophysics. doi.org/10.1016/j.abb.2009.08.015

Nikolaidis, M., Margaritelis, N., Paschalis, V., Theodorou, A., Kyparos, A., y Vrabas, I. (2015). Common questions and tentative answers on how to asses oxidative stress after antioxidant supplementation and exercise. In M. Lamprecht (Ed.), Antioxidants in sports nutrition (1st ed., pp. 221-246). Boca Raton: CRC Press.

Nordin, T. C., Done, A. J., y Traustadóttir, T. (2014). Acute exercise increases resistance to oxidative stress in young but not older adults. Age, 36, 1-9. doi.org/10.1007/ s11357-014-9727-z

Pall, M. L., y Levine, S. (2015). Nrf2, a master regulator of detoxification and also antioxidant, anti-inflammatory and other cytoprotective mechanisms, is raised by health promoting factors Nrf2. Acta Physiologica Sinica, 67(1), 1-18. doi.org/10.13294/j.aps.2015.0001

Parker, L., Stepto, N. K., Shaw, C. S., SerpieIlo, F. R., Anderson, M., Hare, D. L., y Levinger, I. (2016). Acute High-Intensity Interval Exercise-Induced Redox Signaling Is Associated with Enhanced Insulin Sensitivity in Obese Middle-Aged Men. Frontiers in Physiology | Www.Frontiersin.Org, 1(411), 1-11. doi.org/10.3389/fphys.2016.00411

Pérez-Ruiz, M., Córdova-Martínez, A., López-Chicharro, J., y Lucía-Mulas, A. (2006). Respuestas y adaptaciones hematológicas. In Fisiología del ejercicio (3era ed., pp. 281-320). Buenos Aires: Panamericana.

Perl, A. (2015). mTOR activation is a biomarker and a central pathway to autoimmune disorders, cancer, obesity, and aging. Ann NY Acad Sci, 1346(1), 
33-44. doi.org/10.1111/nyas.12756

Powers, S. K., Duarte, J., Kavazis, A. N., Talbert, E. E., y Powers, S. K. (2009). Reactive oxygen species are signaIling molecules for skeletal muscle adaptation. Exp Physiol, 95, 1-9. doi. org/10.1113/expphysiol.2009.050526 Powers, S. K., Talbert, E. E., Adhihetty, P. J., y Powers, S. K. (2011). Reactive oxygen and nitrogen species as intracellular signals in skeletal muscle. J Physiol, 589, 2129-2138. doi. org/10.1113/jphysiol.2010.201327

Pruss-Ustun, A., Wolf, J., Corvalán, C., Bos, R., y Neira, M. (2016). Preventing disease through healthy environments. Francia. Recuperado de http:// apps.who.int/iris/bitstream/handle/10665/204585/9789241565196_ eng.pdf; jsessionid = A1A1FB4547A8F4E9CAEEBAA253C2E217?sequen$\mathrm{ce}=1$

Radak, Z., Taylor, A. W., Ohno, H., y Goto, S. (2001). Adaptation to exercise-induced oxidative stress: from muscle to brain. Exercise Immunology Review, 7, 90-107. Recuperado de https://pubmed.ncbi.nlm.nih. gov/11579750/

Rivera-Brown, A. M., \& Frontera, W. R. (2012). Principles of Exercise Physiology: Responses to Acute Exercise and Long-term Adaptations to Training. PM\&R, 4(11), 797-804. doi.org/10.1016/j.pmrj.2012.10.007

Sakurai, T., Ogasawara, J., Shirato, K., Izawa, T., Oh-Ishi, S., Ishibashi, Y., ... Kizaki, T. (2017). Exercise Training Attenuates the Dysregulated Expression of Adipokines and Oxidative Stress in White Adipose Tissue. Oxidative Me- dicine and Cellular Longevity, 2017, 1-12. doi.org/10.1155/2017/9410954 Schieber, M., y Chandel, N. S. (2014). ROS function in redox signaling and oxidative stress. Current Biology, 24(10), R453-R462. doi.org/10.1016/j. cub.2014.03.034

Taylor, J. K., Esco, M. R., Qian, L., Dugan, K., y Jones, K. (2015). A single session of aerobic exercise influences paraoxonase 1 activity and concentration. Retos: Nuevas Tendencias En Educación Física, Deporte y Recreación, ISSN 1579-1726, No. 27, 2015, Págs. 222-225, 27, 222-225. Recuperado de https://dialnet.unirioja.es/ servlet/articulo? codigo $=5407748$

Tebay, L. E., Robertson, H., Durant, S. T., Vitale, S. R., Penning, T. M., Dinkova-Kostova, A. T., y Hayes, J. D. (2015). Mechanisms of activation of the transcription factor Nrf2 by redox stressors, nutrient cues, and energy status and the pathways through which it attenuates degenerative disease HHS Public Access. Free Radic Biol Med, 88, 108-146. doi.org/10.1016/j.freeradbiomed.2015.06.021

Traustadóttir, T., Davies, S. S., Su, Y., Choi, L., Brown-Borg, H. M., Jackson, L., .. Brown-Borg, H. M. (2012). Oxidative stress in older adults: effects of physical fitness. Age, 34, 969-982. doi.org/10.1007/s11357-011-9277-6

Trayhurn, P., Wang, B., y Wood, I. S. (2018). Horizons in Nutritional Science Hypoxia in adipose tissue: a basis for the dysregulation of tissue function in obesity? doi.org/10.1017/ S0007114508971282 
Viada Pupo, E., Robles, L. G., Reyna, I., y Marrero, C. (2017). Estrés oxidativo. Ccm, (1), 171-186. Recuperado de http://scielo. sld.cu/pdf/ccm/v21n1/ccm14117.pdf

Wang, L., Mascher, H., Psilander, N., Blomstrand, E., y Sahlin, K. (2011). Resistance exercise enhances the molecular signaling of mitochondrial biogenesis induced by endurance exercise in human skeletal muscle. J Appl Physiol, 111, 1335-1344. doi.org/10.1152/japplphysiol.00086.2011.-Combining

Webb, R., Hughes, M., Thomas, A., y Morris, K. (2017). The Ability of Exercise-Associated Oxidative Stress to Trigger Redox-Sensitive Signalling Responses. Antioxidants, 6(3), 63. doi.org/10.3390/ antiox6030063 